\title{
Inflammatory mediators: Parallels between cancer biology and stem cell therapy
}

\author{
Shyam A Patel ${ }^{1,2,3}$ \\ Andrew C Heinrich ${ }^{2,3}$ \\ Bobby Y Reddy ${ }^{2}$ \\ Pranela Rameshwar ${ }^{2}$ \\ 'Graduate School of Biomedical \\ Sciences, University of Medicine \\ and Dentistry of New Jersey, Newark, \\ NJ, USA; ${ }^{2}$ Department of Medicine - \\ Division of Hematology/Oncology, \\ New Jersey Medical School, University \\ of Medicine and Dentistry of New \\ Jersey, Newark, NJ, USA; ${ }^{3}$ These \\ authors contributed equally \\ to this work
}

\begin{abstract}
Inflammation encompasses diverse molecular pathways, and it is intertwined with a wide array of biological processes. Recently, there has been an upsurge of interest in the interactions between mediators of inflammation and other cells such as stem cells and cancer cells. Since tissue injuries are associated with the release of inflammatory mediators, it would be difficult to address this subject without considering the implications of their systemic effects. In this review, we discuss the effects of inflammatory reactions on stem cells and extrapolate on information pertaining to cancer biology. The discussion focuses on integrins and cytokines, and identifies the transcription factor, nuclear factor-kappa B (NFKB) as central to the inflammatory response. Since stem cell therapy has been proposed for type II diabetes mellitus, metabolic syndrome, pulmonary edema, these disorders are used as examples to discuss the roles of inflammatory mediators. We propose prospects for future research on targeting the NFKB signaling pathway. Finally, we explore the bridge between inflammation and stem cells, including neural stem cells and adult stem cells from the bone marrow. The implications of mesenchymal stem cells in regenerative medicine as pertaining to inflammation are vast based on their anti-inflammatory and immunosuppressive effects. Such features of stem cells offer great potential for therapy in graft-versus-host disease, conditions with a significant inflammatory component, and tissue regeneration.
\end{abstract}

Keywords: mesenchymal stem cells, cancer, cytokines, NF kappa B

\section{Inflammation in tumorigenesis}

As early as the 1800s, scientists have proposed a relationship between cancer and chronic inflammation. ${ }^{1,2}$ This connection is based on the observation of uncontrolled cell proliferation at sites of inflammation and the observation of inflammatory cells at regions of the cancer. ${ }^{1}$ Currently, there is resurgence of interests in the relationship between inflammation and cancer. Epidemiological studies confirmed that chronic inflammation renders individuals more susceptible to cancer. ${ }^{3}$ Studies now suggest that nearly one of five cancer deaths is due to malignancies triggered by chronic inflammation. ${ }^{1}$ Additionally, chronic inflammation has been linked to the proliferation and metastasis of tumors. ${ }^{3}$ Therefore, it could be surmised that suppression of chronic inflammation could lead to a decrease in tumorigenesis, hence a decrease in cancer-related fatality.

The etiology of cancer is complex, although inflammation appears to be a recurring theme when this topic is discussed. For example, carcinogens in foods can trigger inflammation followed by cancer. ${ }^{4}$ In the case of malignancy of the prostate, the production of interleukin-6 (IL-6) and other inflammatory mediators may confer a survival advantage to local stem cells, which has been proposed as a method to facilitate tumorigenesis. ${ }^{4}$ Autocrine signaling loops may cause initiation of tumors, and perhaps cause dysfunction in the stem cells. ${ }^{4}$ Other reports have implicated autoimmune conditions and infections in cancer development. ${ }^{1}$ Current knowledge on 
inflammation-induced carcinogenesis is limited but the widespread implications of this phenomenon on human health warrant further studies.

The inflammatory response can be triggered by pathological conditions as such as hypoxia-ischemia. This pathological state in newborns has been shown to elicit a wide array of apoptotic responses. ${ }^{5}$ This information, when combined with the involvement of cytokines, could extrapolate to prospects on cell death and protection of tumors. ${ }^{5}$ During perinatal distress, resuscitation of hypoxic-ischemic conditions with $100 \%$ oxygen can result in shuttling of the Bax channel to various subcellular locations, leading to activation IL- $1 \beta$ and the inflammatory response. ${ }^{5}$ Calpain and Bax levels in the endoplasmic reticulum are also increased, further supporting a role for cytokines in the biology of programmed cell death, and when extrapolated, in cancer cells. ${ }^{5}$

\section{Integrins function in cancer development}

Among the key molecules involved in the inflammatory response are the integrins. ${ }^{6}$ Integrins are members of the cell adhesion molecule family. This family of molecules mediates interactions between cells and the extracellular matrix to facilitate cell migration, adhesion, differentiation, proliferation, angiogenesis, survival, and inflammation. ${ }^{7}$ The integrins have roles in signal transduction by cytokine receptors, G-protein-coupled receptors (GPCRs), and receptor tyrosine kinases (RTKs). ${ }^{8}$ In the neutrophil response to acute inflammation, inflammatory mediators from primary granules include heparin-binding protein, which leads to macrophage activation through $\beta 2$-integrin. ${ }^{6}$ In Gram-negative infection, $\beta 2$-integrin may function as the lipopolysaccharide receptor. ${ }^{9}$ However, it should be noted that roles of integrins are not limited to inflammation. Since the integrins have been implicated in cancer biology, it has been proposed that integrin blockers may synergize with chemotherapy and radiation for treatment of malignancies. ${ }^{7}$

The bridge between cancer and integrins is evident from the observation of anoikis, defined as apoptosis following loss of cell adhesion. ${ }^{10}$ Integrins are responsible for anchoragedependent cell survival, and, along with cadherins, they prevent cancer development. ${ }^{11}$ Anoikis is vital for maintenance of tissue integrity because it prevents defective cells from replicating. ${ }^{12}$ Without the ability to adhere to surrounding surface, cells could travel and proliferate in inappropriate regions. A central property of cancer cells is the ability to avoid anchorage and to proliferate in the absence of integrin and cadherin expression. ${ }^{12}$
In untransformed cells, integrin-induced adhesion leads to cell cycle progression, specifically throughout the G1 phase. $^{7}$ Adhesion molecules also regulate entry into S phase, as integrins interact with cyclin E/cdk2. ${ }^{13}$ Integrins and cell signaling receptors cooperate to modulate cell cycle progression by regulating cyclin D1 expression at the transcriptional and post-translational levels. ${ }^{7}$ These findings suggest that the mechanisms by which cell anchorage molecules regulate cell function should be explored in more detail considering the significance in different pathological conditions, including tumorigenesis.

As the cancer stem cell hypothesis gains acceptance, it is important to understand how the molecules central to cancer development are involved in stem cell biology. Such an understanding might lead to the identification of the elusive cancer stem cells. Embryonic stem cells lacking $\beta 1$-integrin fail to differentiate, substantiating a role for integrins in the differentiation of pluripotent cells. ${ }^{14}$ Conversely, $\beta 1$-integrin deficiency in neuronal tissue facilitates cell differentiation. ${ }^{7}$ A specific oncogenic $\beta 1$-integrin mutation appears to facilitate ligand binding and to hinder differentiation of skin cells. ${ }^{7}$ We can therefore speculate that these ligand-binding events can promote development of high-grade malignancies.

\section{Pro-inflammatory cytokines have diverse roles in cancer}

The prospect of cytokine involvement in malignancy has received attention. This section discusses two cytokines: IL-2 and IL-21 due to their similarities and sharing of the $\gamma$-chain subunit of the receptor. ${ }^{15}$ Despite this similarity, these two cytokines have opposing effects. IL-2, for example, promotes the maturation of cytotoxic $\mathrm{T}$ lymphocytes to granzyme B-expressing cells while IL-21 inhibits these processes. ${ }^{15}$ IL-2 also promotes the development of regulatory $\mathrm{T}\left(\mathrm{T}_{\text {reg }}\right)$ cells and aids in the activation and expansion of $\mathrm{T}$ cells that can lyse tumor cells. ${ }^{15}$ These experimental findings have led to the application of IL-2 in anti-cancer therapies. ${ }^{16}$ Investigators have suggested that the antagonistic relationship between IL-2 and IL-21 can be vital to the use of T cells for immunotherapy for cancer, and perhaps other immunemediated disorders. ${ }^{15}$

Another relevant inflammatory cytokine is tumor necrosis factor- $\alpha$ (TNF- $\alpha$ ), a macrophage pro-inflammatory cytokine that appears to have a bimodal effect on tumorigenesis. ${ }^{17}$ Whereas it facilitates proliferation of tumor cells such as those of the lung, it also exerts pro-apoptotic effects. ${ }^{18}$ The oncogenic effects are evident in that TNF- $\alpha$ 
facilitates proliferation of cells in breast cancer and renal cell carcinoma. ${ }^{17,19}$ These findings may explain why TNF- $\alpha$ - and TNF- $\alpha$-receptor-deficient mice are less susceptible to carcinogenesis. ${ }^{17}$ On the other end of the spectrum, TNF- $\alpha$ therapy for treatment of cancer has been investigated. Although groups of low-invasive carcinoma cells have been identified as TNF- $\alpha$-sensitive, others have shown resistance. ${ }^{18}$ Investigations into the mechanisms of breast cancer resistance to TNF- $\alpha$ therapy are warranted if the future of cancer therapy includes this valuable cytokine. In any case, the specific delivery of TNF- $\alpha$ is critical in order for it to be successful in the clinic, as TNF- $\alpha$ is toxic to normal body functions. ${ }^{18}$

\section{Chemokines}

Chemokines are a family of small molecules that facilitate cell movement via adhesion and migration. ${ }^{20}$ They alter the functions of target cells by paracrine, endocrine, and autocrine stimulation. ${ }^{21}$ Chemokine receptors, such as CXCR4, in malignant cells have been shown to promote cell proliferation, migration, and maintenance. Such events contribute to metastasis, such as the role of one chemokine family, stromal cell-derived factor-1 $\alpha$ (SDF-1 $\alpha)$ in breast cancer invasion and metastasis to bone marrow. ${ }^{21,22}$

SDF- $1 \alpha$ and its receptor CXCR4 are crucial to migration of breast cancer cells to sites of distant metastasis, such as bone marrow, lung, liver, and brain..$^{22}$ Expression of SDF-1 $\alpha$ in breast cancer cells assists in cellular and vascular proliferation. ${ }^{21}$ These findings are consistent with studies demonstrating that plasma levels of SDF- $1 \alpha$ are higher in patients with breast cancer than healthy individuals. ${ }^{22}$ On the contrary, this chemokine/receptor pair appears to promote quiescence of malignant breast cells in the marrow cavity. ${ }^{22}$ In contrast to carcinoma, normal breast tissue expresses low levels of CXCR4, substantiating the cancer-inducing nature of the SDF- $1 \alpha /$ CXCR 4 axis. In addition to SDF- $1 \alpha$, another member of the chemokine family, monocyte chemotactic protein-1 (MCP-1), is particularly important to breast cancer. ${ }^{23}$ MCP-1 is secreted by various immune cell types and plays an integral role in advanced breast cancer progression. ${ }^{23}$ It functions in molecular interactions with the microenvironment. ${ }^{23}$ MCP-1 also recruits immune cells, such as macrophages, to sites of inflammation. ${ }^{21}$ The link to cancer is evident in studies demonstrating high levels of MCP-1 secretion by tumor cells. In patients with acute lymphoblastic leukemia, for example, MCP-1 levels are significantly elevated during treatment, suggesting a regulatory mechanism in this cancer. ${ }^{24}$

Inflammation in the brain is partly regulated by the SDF$1 \alpha /$ CXCR 4 axis, the same interaction that governs breast cancer metastasis to the bone marrow. ${ }^{25}$ Normally, SDF-1 $\alpha$ is expressed by bone marrow stromal cells, and CXCR4 is expressed on the surface of breast cancer cells. ${ }^{22}$ The interaction between these two molecules has been suggested to facilitate dormancy of breast cancer in the bone marrow. SDF-1 $\alpha$ knockdown in breast cancer cells leads to reduced efficiency of cancer cell entry into bone marrow. ${ }^{22}$ Clearly, inflammation plays an essential role in various types of stem cell events.

It is clear that the role of chemokines in breast cancer is great and the potential for chemokine-targeted treatment is very appealing. Interestingly, evidence suggests that the expression of the chemokines SDF- $1 \alpha$ and MCP-1 can be regulated by endogenous hormones. ${ }^{21}$ The prospect of regulation by endogenous hormones suggests a role for supplemental hormones as well. Therefore, the hormonal suppression of these metastasis-promoting chemokines may be one potential breast cancer treatment method.

\section{NFkB transcription factor in inflammation and tumorigenesis}

The transcription factor NFKB has been of interest for inflammatory-mediated responses, mostly because several cytokines cause the activation of this transcription factor. ${ }^{26}$ The

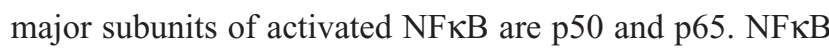
is activated when the inhibitor kappaB (IKB) is phosphorylated by IKB kinase (IKK). ${ }^{26}$ Its phosphorylation permits translocation to the nucleus and transcriptional activation of several genes. ${ }^{26}$ The functions of NFKB span diverse cellular processes, including adhesion, proliferation, apoptosis, differentiation, immune regulation, and angiogenesis. ${ }^{27}$ The most common conditions associated with NFKB signaling are chronic inflammatory diseases, such as rheumatoid arthritis, psoriatic arthritis, celiac disease, ulcerative colitis, and Crohn's disease. ${ }^{27}$

The NFKB pathway may be central to the process of atherosclerosis with regards to inflammation of the vascular endothelium. ${ }^{28}$ Lymphotoxin family members, including TNF- $\alpha$, appear to employ NFKB signaling in vessel injury. ${ }^{28}$ Lymphotoxin- $\alpha$, for example, increases VCAM1 expression on endothelial cells in an NFKB-dependent manner. ${ }^{28}$ These findings are consistent with demonstrations of the rescue of NFKB effector function by TNF- $\alpha .{ }^{26}$ The precise mechanism of TNF- $\alpha$-mediated activation of NFKB signaling may be through the inhibition of IкB. ${ }^{29}$

The role of NFKB in pulmonary edema has recently been investigated. NFKB expression increased 13 -fold during periods of hypoxia in patients with pulmonary edema. ${ }^{30}$ 
The DNA-binding function of $\mathrm{NF} \kappa \mathrm{B}$ was also increased, suggesting that NFKB activity may be a response to oxidative stress produced by free radical generation under hypoxic conditions. ${ }^{30}$ In addition to NFKB upregulation, levels of other pro-inflammatory cytokines such as TNF- $\alpha$ and acute phase reactants such as IL-1 and IL-6 increased. ${ }^{30}$ Treatment with curcumin, an NFKB antagonist, prevented the inflammatory response associated with hypoxia, specifically vascular leakage in the lungs. ${ }^{30}$ The role of NFKB in pulmonary tissue is not completely understood at this point, yet it appears to be vital in hypoxia-induced stress.

The implications of NFKB in hypoxia are not limited to pulmonary edema, but encompass other pathological processes. NFKB may mediate the response to ischemic pre-conditioning and cardioprotection of the heart. ${ }^{31}$ The activation of the NFKB pathway followed by $\mathrm{K}_{\text {ATP }}$ channel opening mediates cardio- and renal-protective events. ${ }^{31}$

In addition to its role in these processes, NFKB may be an important mediator in the disease processes of type II diabetes mellitus and the metabolic syndrome. ${ }^{32}$ Reactive oxygen species and reactive nitrogen species activate $\mathrm{NF} \kappa \mathrm{B}$ and subsequent transcription of TNF- $\alpha$. This series of events has been linked to insulin resistance in skeletal muscle, thereby suggesting a role for $\mathrm{NF} \kappa \mathrm{B}$ in diabetes and metabolic syndrome. ${ }^{32}$

Although much attention regarding NF $\kappa \mathrm{B}$ has focused on inflammatory states, the current upsurge of interest in stem cell biology has led to studies of the function of NFKB in stem cells of the bone marrow. NFKB has been suggested to regulate secretion of growth factors and cytokines in adult and neonatal stem cells from the bone marrow environment. ${ }^{22}$ Vascular endothelial growth factor (VEGF) was decreased upon IKK inhibition in adult stem cells. ${ }^{26}$ This effect was completely reversed by TNF- $\alpha$, a member of the lymphotoxin family. ${ }^{26,28}$ Furthermore, IL-6 levels were decreased upon $\mathrm{NF} \kappa \mathrm{B}$ inhibition in both adult and neonatal stem cells. ${ }^{26}$ These findings suggest that NFKB is pivotal in production of VEGF by stem cells. Aside from bone marrow stem cells, neural stem cells also employ the NFKB pathway, which facilitates migration, proliferation, and differentiation of these stem cells..$^{33}$ Although these findings have demonstrated a role of NFKB in stem cell biology, our knowledge in this area is still incomplete and therefore under investigation by our laboratory.

Recent literature has pointed to the role of NFKB in malignant processes, as numerous cancers demonstrate dysregulation of the NFKB pathway. ${ }^{34}$ The metastatic oral squamous cell carcinoma line HSC3, for example, showed significantly higher levels of the p65 subunit of NFкB. ${ }^{34}$ Furthermore, TNF- $\alpha$ signaling depends on NFkB in the malignant keratinocytes of squamous cell carcinoma of the skin. ${ }^{35}$ In gastric carcinoma, NF $\kappa \mathrm{B}$ exerts an anti-apoptotic effect via upregulation of the survivin gene. ${ }^{36}$

A multitude of other pathological processes involve NFKB but have not been thoroughly investigated thus far. For example, an anti-apoptotic function of NFKB has been suggested for osteoclasts. Transforming growth factor- $\beta$ (TGF- $\beta$ ) utilizes the NFKB pathway, in addition to other pathways, to prevent programmed cell death of osteoclasts. Multiple pathways, including the TAK1/MEK pathway, are involved in the NFKB-mediated anti-apoptotic effect. ${ }^{37} \mathrm{NF \kappa B}$ appears to play a role in regulation of spermatogenesis. ${ }^{38} \mathrm{It}$ appears to regulate the junctional complexes between Sertoli cells and spermatids. ${ }^{39}$ Clearly, the implications of NFkB are vast, and future studies on this master regulator hold great potential in treatment of disease processes.

\section{Stem cells and inflammation}

The processes underlying tissue injury and repair following inflammation have been studied, and the evidence suggests stem cell involvement. ${ }^{40}$ The induction of an inflammatory response by IL-1 in the lacrimal gland has provided insights into mechanisms of inflammation on stem cells. ${ }^{40}$ In this study, acinar cells of the lacrimal gland underwent apoptosis and autophagy. ${ }^{40}$ Interestingly, the acinar cells expressed the stem cell marker nestin and the cell proliferation marker Ki67. ${ }^{40}$ These studies suggest that lacrimal cells may revert to a stem-like phenotype upon induction of inflammation, or they may contain stem cells that function to regenerate the parenchyma of the organ.

The effects of inflammation on neural stem cell activity is interesting and currently under investigation. Inflammation of the brain has been suggested to lead to neurological diseases including Parkinson's disease, Alzheimer disease, and depression..$^{41}$ Throughout the process of inflammation, the generation of neuronal cells of the adult brain is affected, suggesting that neuroinflammation may offer therapeutic potential for neurodegenerative diseases. ${ }^{41}$

Neural progenitors and stem cells have been postulated to promote neuroprotection via regulation of the immune response. ${ }^{42}$ Stem cells can interact with the host immune system to facilitate host recovery. ${ }^{41}$ The reciprocal protective relationship between stem cells and inflammatory responses is evident from the hypothesis that IL-6 and other inflammatory mediators can facilitate survival of stem cells of the prostate epithelium in patients with prostate cancer. ${ }^{4,43}$ 
The process of inflammation can adversely affect neural stem cell grafts because neural progenitors and stem cells express cell surface receptors for products of the inflammatory response. ${ }^{41}$ Thus, the precise time at which stem cell transplants are administered may be important in bypassing inflammation. $^{41}$

\section{Mesenchymal stem cell therapy for inflammatory-mediated disorders}

Mesenchymal stem cells (MSCs) offer considerable therapeutic value in modulating inflammation and graft rejection. ${ }^{44}$ It is their anti-inflammatory properties rather than their potential as stem cells that warrant their first clinical application. ${ }^{45}$ One of the most serious concerns regarding bone marrow transplantation is graft-versus-host-disease (GVHD), which is a pathology characterized by an immune response of the maturing donor lymphocytes against antigens of the recipient. ${ }^{45}$ GVHD induces systemic inflammation and often results in death of the recipient. ${ }^{45}$ The immune properties of MSCs confer them with pharmacological potential in the regulation of conditions such as GVHD and other inflammatory processes. ${ }^{46}$ In addition to GVHD, MSC therapy has implications in Charcot-Marie-Tooth disease, an inherited neuropathy characterized by demyelination of peripheral nerves. ${ }^{45}$

As for the bimodal property of MSC behavior, it appears that the level of expression is key. An abundance of MSCs leads to immunosuppressive behavior, whereas sparse MSC presence induces immunostimulation. ${ }^{47} \mathrm{MSC}$ s have the ability to suppress immune reactions via activating $\mathrm{T}_{\text {reg }}$ cells and preventing the maturation of functional dendritic cells. ${ }^{48,49}$ This is of particular relevance to hematopoietic stem cell (HSC) transplants because T-cell-derived inflammatory cytokines such TNF- $\alpha$ and IFN- $\gamma$ suppress hematopoiesis. ${ }^{45}$ Therefore, MSCs transplanted along with HSCs can guard them from destruction by inflammatory cytokines. Furthermore, research has shown that MSCs are capable of producing immune suppressive factors such as HLA-G, prostaglandin E2, IL-10, and TGF- $\beta$, which strengthens their anti-inflammatory properties. ${ }^{45}$ Currently, phase III clinical trials are in progress for the use of MSCs in suppressing inflammatory immune responses associated with GVHD, Crohn's disease, and Charcot-Marie-Tooth syndrome. ${ }^{45}$

\section{Obstacles to stem cell therapy}

We have described numerous benefits of stem cell therapy thus far. Intravenous MSC treatments have already been shown to decrease expression of numerous pro-inflammatory cytokines such as IFN- $\gamma$ and TNF- $\alpha .{ }^{29}$ Due to the correlation between the inflammatory process and carcinogenesis, tumorigenesis, angiogenesis, and metastasis, it is clear that the inhibition of the autoimmune response could be a successful anti-cancer measure. While the utilization of their immunosuppressive properties is a promising theory, MSCs present two major obstacles: their oncogenic properties and their senescenceinduced degradation. ${ }^{51}$ MSCs have been shown to stimulate the immune response in some instances, which, due to the connection between inflammation and carcinogenesis, is a serious concern. As for senescence, while it is still a lively debate amongst scientists, there are many who have found evidence that shows that the capabilities and effectiveness of MSCs decrease as they age. ${ }^{51}$ While oncogenesis is the greater concern, both of these complications must be resolved before MSC treatment for cancer is widespread.

\section{Conclusions}

As discussed here, the outlook on cancer biology and stem cell therapy is not complete without considering inflammatory events. Cytokines could be important in the battle against malignancies of the breast and other tissues, either directly or by studying their roles and identifying targets. IL-2-mediated tumor cell lysis implies the significance of cell-based therapy in carcinogenesis. Integrin antagonists are another interesting tool as they regulate differentiation, survival, and angiogenesis, thereby making their presence essential in the prevention of cancer. While a treatment of integrin antagonists alone may not be in the immediate future, evidence shows that they may greatly enhance the effects of chemotherapy and radiotherapy. As with any proposed treatment, however, integrin antagonist treatment has a major difficulty. Targeting the specific integrins of breast cancer cells would prove to be an extraordinarily difficult task, and before such specificity can be guaranteed, this treatment may be dangerous to the patient. Overall, the role of MSCs may extend to conditions with significant inflammatory and autoimmune components. In any case, science must be aware of the obstacles to stem cell therapy before these cells can be administered in a clinical setting.

\section{Disclosure}

This work was funded by the Department of Defense and New Jersey Cancer Commission.

\section{References}

1. Mantovani A, Allavena P, Sica A, Balkwill F. Cancer-related inflammation. Nature. 2008;454:436-444.

2. Jung YJ, Isaacs JS, Lee S, et al. IL-1beta-mediated up-regulation of HIF1alpha via an NFkappaB/COX-2 pathway identifies HIF-1 as a critical link between inflammation and oncogenesis. FASEB J. 2003;17:2115-2117. 
3. Balkwill F, Charles KA, Mantovani A. Smoldering and polarized inflammation in the initiation and promotion of malignant disease. Cancer Cell. 2005;7:211-217.

4. Maitland NJ, Collins AT. Inflammation as the primary aetiological agent of human prostate cancer: A stem cell connection? J Cell Biochem. 2008; 105:931-939.

5. Gill MB, Bockhorst K, Narayana P, Perez-Polo JR. Bax shuttling after neonatal hypoxia-ischemia: Hyperoxia effects. $J$ Neurosci Res. 2008;86:3584-3604.

6. Soehnlein O, Kai-Larsen Y, Frithiof R, et al. Neutrophil primary granule proteins HBP and HNP1-3 boost bacterial phagocytosis by human and murine macrophages. J Clin Invest. 2008;118:3491-3502.

7. Huveneers S, Truong H, Danen HJ. Integrins: signaling, disease, and therapy. Int J Radiat Biol. 2007;83:743-751.

8. Short SM, Boyer JL, Juliano RL. Integrins regulate the linkage between upstream and downstream events in $\mathrm{G}$ protein-coupled receptor signaling to mitogen-activated protein kinase. J Biol Chem. 2000;275: 12970-12977.

9. Ebdrup L, Krog J, Granfeldt A, Tønnesen E, Hokland M. Dynamic expression of the signal regulatory protein alpha and CD18 on porcine PBMC during acute endotoxaemia. Scand J Immunol. 2008;68:430-437.

10. Marastoni S, Ligresti G, Lorenzon E, Colombatti A, Mongiat M: Extracellular matrix: a matter of life and death. Connect Tissue Res. 2008;49:203-206.

11. Haraguchi M, Okubo T, Miyashita Y, et al. Snail regulates cell-matrix adhesion by regulation of the expression of integrins and basement membrane proteins. J Biol Chem. 2008;283:23514-23523.

12. Chiarugi P, Giannoni E: Anoikis. A necessary death program for anchorage-dependent cells. Biochem Pharmacol. 2008;76:1352-1364.

13. Klekotka PA, Santoro SA, Ho A, Dowdy SF, Zutter MM: Mammary epithelial cell-cycle progression via the alpha(2)beta(1) integrin: unique and synergistic roles of the alpha(2) cytoplasmic domain. Am J Pathol. 2001;159:983-992.

14. Fassler R, Rohwedel J, Maltsev V, et al. Differentiation and integrity of cardiac muscle cells are impaired in the absence of beta 1 integrin. J Cell Sci. 1996;109(Pt 13):2989-2999.

15. Hinrichs CS, Spolski R, Paulos CM, et al. IL-2 and IL-21 confer opposing differentiation programs to CD8+ T cells for adoptive immunotherapy. Blood. 2008;111:5268-5269.

16. Papaetis GS, Karapanagiotou LM, Pandha H, Syrigos KN. Targeted therapy for advanced renal cell cancer: cytokines and beyond. Curr Pharm Des. 2008;14:2229-2251.

17. Kundu JK, Surh YJ. Inflammation: Gearing the journey to cancer. Mutat Res. 2008;659:15-30.

18. Wang Z, Kishimoto H, Bhat-Nakshatri P, Crean C, Nakshatri H. TNFalpha resistance in MCF-7 breast cancer cells is associated with altered subcellular localization of p21CIP1 and p27KIP1. Cell Death Differ. 2005;12:98-100.

19. Falkensammer C, Jöhrer K, Gander H, et al. IL-4 inhibits the TNF-alpha induced proliferation of renal cell carcinoma (RCC) and cooperates with TNF-alpha to induce apoptotic and cytokine responses by RCC: implications for antitumor immune responses. Cancer Immunol Immunother. 2006;55:1228-1237.

20. Clahsen T, Schaper F. Interleukin-6 acts in the fashion of a classical chemokine on monocytic cells by inducing integrin activation, cell adhesion, actin polymerization, chemotaxis, and transmigration. J Leukoc Biol. 2008;84:1521-1529.

21. Potter SM, Dwyer RM, Curran CE, et al. Systemic chemokine levels in breast cancer patients and their relationship with circulating menstrual hormones. Breast Cancer Res Treat. 2008 Jun 4 [Epub ahead of print].

22. Moharita AL, Taborga M, Corcoran KE, Bryan M, Patel PS, Rameshwar P. SDF-1 regulation in breast cancer cells contacting bone marrow stroma is critical for normal hematopoiesis. Blood. 2006; 108:3245-3252.

23. Soria G, Yaal-Hahoshen N, Azenshtein E, et al. Concomitant expression of the chemokines RANTES and MCP-1 in human breast cancer: A basis for tumor-promoting interactions. Cytokine. 2008;44:191-200.
24. Eisenkraft A, Keidan I, Bielorai B, Keller N, Toren A, Paret G. MCP-1 in the cerebrospinal fluid of children with acute lymphoblastic leukemia. Leuk Res. 2006;30:1259-1261.

25. Schönemeier B, Kolodziej A, Schulz S, Jacobs S, Hoellt V, Stumm R. Regional and cellular localization of the CXCl12/SDF-1 chemokine receptor CXCR7 in the developing and adult rat brain. J Comp Neurol. 2008;510:207-220.

26. Novotny NM, Markel TA, Crisostomo PR, Meldrum DR. Differential IL-6 and VEGF secretion in adult and neonatal mesenchymal stem cells: Role of NFKB. Cytokine. 2008;43:215-219.

27. Sun XF, Zhang H. NFאB and NFKBI polymorphisms in relation to susceptibility of tumour and other diseases. Histol Histopathol. 2007;22:1387-1398.

28. Suna S, Sakata Y, Sato H, et al. Up-regulation of cell adhesion molecule genes in human endothelial cells stimulated by lymphotoxin alpha: DNA microarray analysis. $J$ Atheroscler Thromb. 2008;15:160-165.

29. Kim HE, Choi SE, Lee SJ, et al. TNF-alpha-induced GSIS inhibition in INS-1 cells is ascribed to a reduction of the glucose-stimulated Ca2+ influx. $J$ Endocrinol. 2008;198:549-560.

30. Sarada SS, Himadri P, Mishra C, Geetali P, Sai Ram M, Ilavazhagan G. Role of oxidative stress and NF $\mathrm{KB}$ in hypoxia induced pulmonary edema. Exp Biol Med. 2008;233:1088-1098.

31. Diwan V, Kant R, Jaggi AS, Singh N, Singh D. Signal mechanism activated by erythropoietin preconditioning and remote renal preconditioninginduced cardioprotection. Mol Cell Biochem. 2008;315(1-2):195-201.

32. Silveira LR, Fiamoncini J, Hirabara SM, et al. Updating the effects of fatty acids on skeletal muscle. J Cell Physiol. 2008;217:1-12.

33. Cimini A, Cerù MP. Emerging roles of peroxisome proliferatoractivated receptors (PPARs) in the regulation of neural stem cells proliferation and differentiation. Stem Cell Rev. 2008;4:293-303.

34. Sasahira T, Kirita T, Oue N, et al. High mobility group box-1-inducible melanoma inhibitory activity is associated with nodal metastasis and lymphangiogenesis in oral squamous cell carcinoma. Cancer Sci. 2008;99:1806-1812.

35. Faurschou A, Gniadecki R. TNF-alpha stimulates Akt by a distinct aPKC-dependent pathway in premalignant keratinocytes. Exp Dermatol. 2008;17:992-997.

36. Fukayama M, Hino R, Uozaki H. Epstein-Barr virus and gastric carcinoma: virus-host interactions leading to carcinoma. Cancer Sci. 2008;99:1726-1733.

37. Gingery A, Bradley EW, Pederson L, Ruan M, Horwood NJ, Oursler MJ. TGF-beta coordinately activates TAK1/MEK/AKT/NFkB and SMAD pathways to promote osteoclast survival. Exp Cell Res. 2008;314: 2725-2738.

38. Shalini S, Bansal MP. Co-operative effect of glutathione depletion and selenium induced oxidative stress on API and NFKB expression in testicular cells in vitro: insights to regulation of spermatogenesis. Biol Res. 2007;40:307-317.

39. Tash JS, Chakrasali R, Jakkaraj SR, et al. Gamendazole, an orally active indazole carboxylic acid male contraceptive agent, targets HSP90AB1 (HSP90BETA) and EEF1A1 (eEF1A), and stimulates Il1a transcription in rat sertoli cells. Biol Reprod. 2008;78:1139-1152.

40. Zoukhri D, Fix A, Alroy J, Kublin CL. Mechanisms of murine lacrimal gland repair after experimentally induced inflammation. Invest Ophthalmol Vis Sci. 2008;49:4399-4406.

41. Taupin P. Adult neurogenesis, neuroinflammation and therapeutic potential of adult neural stem cells. Int J Med Sci. 2008;5:127-132.

42. Pluchino S, Zanotti L, Rossi B, et al. Neurosphere-derived multipotent precursors promote neuroprotection by an immunomodulatory mechanism. Nature. 2005;436(7048):266-271.

43. Birnie R, Bryce SD, Roome C, et al. Gene expression profiling of human prostate cancer stem cells reveals a pro-inflammatory phenotype and the importance of extracellular matrix interactions. Genome Biol. 2008;9(5):R83.

44. Zhou H, Jin Z, Liu J, Yu S, Cui Q, Yi D. Mesenchymal stem cells might be used to induce tolerance in heart transplantation. Med Hypotheses. 2008;70:785-787. 
45. Leal A, Ichim TE, Marleau AM, Lara F, Kaushal S, Riordan NH. Immune effects of mesenchymal stem cells: Implications for CharcotMarie-Tooth disease. Cell Immunity. 2008;253(1-2):11-15.

46. Augello A, Tasso R, Negrini SM, Cancedda R, Pennesi G. Cell therapy using allogeneic bone marrow mesenchymal stem cells prevents tissue damage in collagen-induced arthritis. Arthritis Rheum. 2007;56:1175-1186

47. Patel SA, Sherman L, Munoz J, Rameshwar P. Immunological properties of mesenchymal stem cells and clinical implications. Arch Immunol Ther Exp. 2008;56:1-8.

48. Selmani Z, Naji A, Zidi I, et al. Human leukocyte antigen-G5 secretion by human mesenchymal stem cells is required to suppress $\mathrm{T}$ lymphocyte and natural killer function and to induce CD4 + CD25highFOXP3+ regulatory T cells. Stem Cells. 2008;26:212-222.
49. Chen L, Zhang W, Yue H, et al. Effects of human mesenchymal stem cells on the differentiation of dendritic cells from CD34+ cells. Stem Cells Dev. 2007;16:719-731.

50. Kim YJ, Park HJ, Lee G, et al. Neuroprotective effects of human mesenchymal stem cells on dopaminergic neurons through anti-inflammatory action. Glia. 2009;57:13-23.

51. Stolzing A, Jones E, McGonagle D, Scutt A. Age-related changes in human bone marrow-derived mesenchymal stem cells: consequences for cell therapies. Mech Aging Dev. 2008;129:163-173. 
